\title{
Mechanical Enhancement of Bioinspired Polydopamine Nanocoatings
}

Katerina G. Malollari1t, Peyman Delparastan2t, Caroline Sobek3, Shraddha J. Vachhani4, Tanner D. Fink5, R. Helen Zha5, and Phillip B. Messersmith2,6,7*

tThese authors contributed equally

1 Department of Mechanical Engineering, University of California, Berkeley, CA, USA

2 Department of Materials Science and Engineering, University of California, Berkeley, CA, USA

${ }_{3}$ College of Chemistry, University of California, Berkeley, CA, USA

4 Bruker Nano Surfaces, Minneapolis, MN, USA

5 Department of Chemical \& Biological Engineering, Rensselaer Polytechnic Institute, Troy, NY, USA

${ }_{6}$ Department of Bioengineering, University of California, Berkeley, CA, USA

7 Materials Sciences Division, Lawrence Berkeley National Laboratory, Berkeley, CA, USA

${ }^{*}$ Contact Author:

philm@berkeley.edu

210 Hearst Memorial Mining Building

Department of Materials Science and Engineering

Berkeley, CA, USA 94720-1760

510-643-9631 


\section{Table of Contents:}

1. Materials and Methods

2. Table S1 - Elemental Composition from XPS

3. Figure S1 - Contact Angle

4. Figure S2 - UV-Vis Spectroscopy

5. Figure S3 - Thickness Evaluation after Sonication

6. Figure S4 - GIWAXS on pDA-Coated and Bare $\mathrm{TiO}_{2}$ Substrates

7. Figure S5 - Scratch Resistance - Constant Load

8. Figure S6 - Scratch Resistance - Progressive Load

9. Figure S7 - Elastic Modulus

10. Figure S8 - pDA-Assisted Electroless Metallization 


\section{Materials and Methods}

\subsection{Materials}

Dopamine hydrochloride (99\%, Alfa Aesar), sodium metaperiodate $\left(\mathrm{NalO}_{4}, 98 \%\right.$, Alfa Aesar), bicine (99\%, Alfa Aesar), sodium hydroxide ( $\mathrm{NaOH}$, pellets, Fisher Scientific), Silver Nitrate (99.9\% Alfa Aesar), hydrogen peroxide (35 wt\% $\mathrm{H}_{2} \mathrm{O}_{2}$, Acros Organics), sulfuric acid (96\%, $\mathrm{H}_{2} \mathrm{SO}_{4}$, Fisher Scientific), isopropyl alcohol (99.5\%, VWR Analytical), acetone (Macron Fine Chemicals), and Simple Green (Sunshine Makers, Inc) were used as received. Quartz coverslips were purchased from Ted Pella, Inc. (Redding, CA). Silicon wafers $\left(\mathrm{SiO}_{2}\right)$ with a layer of $100 \mathrm{~nm}$ titanium oxide $\left(\mathrm{TiO}_{2}\right)$ were obtained from University Wafer, Inc. (Boston, MA). Ultrapure (UP) water was obtained by purification of deionized water with a Barnstead Ultrapure Water Purification System (Thermo Fisher Scientific, Waltham, MA) to a resistivity of $18.2 \mathrm{M} \Omega \mathrm{cm}$.

\subsection{Coating Substrates with pDA}

$\mathrm{TiO}_{2}$ substrates were first degreased with a 3:1 (v/v) mixture of water and Simple Green solution by placing into sonication bath for 15 minutes at ambient temperature $\left(\sim 22^{\circ} \mathrm{C}\right)$. The substrates were then rinsed and sonicated sequentially with water, isopropanol, and acetone, each for 15 minutes, and dried under a stream of nitrogen gas. Prior to immersing in dopamine solution, substrates were exposed to a plasma discharge at 100 W for 5 minutes (Harrick Plasma Cleaner, Ithaca, NY, USA). Quartz slides were cleaned by placing into Piranha solution $\left(\mathrm{H}_{2} \mathrm{SO}_{4}: \mathrm{H}_{2} \mathrm{O}_{2} 70: 30 \mathrm{v} / \mathrm{v}\right)$ for 1 hour at ambient temperature and were then rinsed with UP water and dried with nitrogen. The substrates were immersed into $2 \mathrm{mg} / \mathrm{ml}$ dopamine hydrochloride in $100 \mathrm{mM}$ bicine buffer at $\mathrm{pH} 8.5$ for 16 hours (20 hours for quartz slides) with constant shaking. The substrates were removed and rinsed extensively with UP water and dried under a stream of nitrogen gas.

\section{3. pDA-Assisted Electroless Metallization}

pDA coated $\mathrm{TiO}_{2}$ substrates were immersed into $50 \mathrm{mM}$ aqueous silver nitrate solutions for $20 \mathrm{~h}$ at room temperature under constant stirring. Surfaces were washed extensively and dried under a stream of nitrogen gas. 


\section{4. pDA NPs Synthesis}

TGA and TGA-MS measurements were performed on pDA particles. Dopamine hydrochloride $(2 \mathrm{mg} / \mathrm{ml})$ was dissolved in UP water and the $\mathrm{pH}$ was increased to 8.5 by adding $1 \mathrm{M} \mathrm{NaOH}$ solution. The solution was stirred at $400 \mathrm{rpm}$ for 5 hours at $70{ }^{\circ} \mathrm{C}$ and subsequently left at ambient temperature for 3 hours without stirring. Finally, the mixture was filtered with PVDF membrane filters with a pore size of $0.1 \mu \mathrm{m}$ (Durapore VVPP) followed by drying at $50{ }^{\circ} \mathrm{C}$ under vacuum.

\subsection{Thermal and Chemical Treatment of pDA}

pDA-coated substrates and NPs were annealed for 12 hours (72 hours for quartz slides) at $130{ }^{\circ} \mathrm{C}$ under vacuum. Ellipsometry, contact angle, and XPS measurements were performed on the samples after allowing them to equilibrate to room temperature. For the chemical stability test, samples were immersed for 2 hours in $1 \mathrm{M}$ aqueous $\mathrm{NaOH}$ solution and then removed and rinsed with water and dried under nitrogen gas. For chemical stability test under sonication pristine pDA and annealed pDA coated $\mathrm{TiO}_{2}$ were sonicated in an aqueous environment ( $\mathrm{pH} 6.9)$ at high power for 10 minutes.

\subsection{X-Ray Photoelectron Spectroscopy (XPS)}

The surface chemical composition of pristine and annealed pDA coatings on $\mathrm{TiO}_{2}$ substrates was measured using X-ray photoelectron spectroscopy (XPS). XPS analyses were performed on a Perkin Elmer Phi 5600, equipped with a monochromatic Al Ka Xray source operating at $350 \mathrm{~W}$. The neutralizer accessory was used with an emission of $1 \mu \mathrm{A}$ to inhibit charging of the samples. All spectra were calibrated to a C1s peak at 284.8 $\mathrm{eV}$. For each sample measured, multiple survey scans were taken at different positions, with a pass energy of $187.85 \mathrm{eV}$ followed by high resolution spectra of $\mathrm{C} 1 \mathrm{~s}, \mathrm{~N} 1 \mathrm{~s}$, and O1s with a pass energy of $23.50 \mathrm{eV}$. Calibrated spectra were fitted with Gaussian functions using OriginPro software. The minimum number of peaks consistent with the 
best fit were used, while peak position, full width at half maximum, and intensity were also considered during the peak fitting.

\subsection{UV-Vis Spectroscopy}

The UV-Vis absorbance spectra of the pDA-coated quartz slides were taken at a wavelength range of $200-800 \mathrm{~nm}$ using a UV2600 spectrophotometer (Shimadzu Scientific Instruments, Kyoto, Japan).

\subsection{Thermogravimetric Analysis (TGA) / Mass spectrometry (TGA-MS)} Thermogravimetric analyses were conducted using a TA Instruments TGA Q5000 and decomposition trace was collected under a flow rate of $25 \mathrm{~mL} / \mathrm{min} \mathrm{N}_{2}$ with a temperature ramp rate of $1.5^{\circ} \mathrm{C} / \mathrm{min}$. TGA-MS performed over a $\mathrm{m} / \mathrm{z}$ range of 15 to 120 under nitrogen flow using a Perkin Elmer Pyris 1 TGA paired with a Hiden Analytical HPR20 Sampling System.

\subsection{Ellipsometry}

The thickness of the pristine and annealed pDA deposited on the $\mathrm{TiO}_{2}$ substrates was measured with a spectroscopic ellipsometer (M-2000V, J.A. Woollam, USA). The spectra were fitted with multilayer slab models using CompleteEase software. The $\mathrm{TiO}_{2}$ layer was fitted with a Cauchy model immediately prior to immersion in the dopamine solution whereas pDA deposits on the $\mathrm{TiO}_{2}$ were fitted with a $\mathrm{B}$-spline model. For the liquid cell measurements, thickness of the dry coatings in an empty, aligned $500-\mu \mathrm{L}$ liquid cell were determined at an incidence angle of $70^{\circ}$. Deionized water (room temperature) was then introduced into the cell and the thickness was measured at half-hour increments for 6 hours. Best-fit for coating thicknesses and optical constants were determined from analyzing data with the CompleteEASE software.

\subsection{Contact Angle}

Water contact angle of the coatings on $\mathrm{TiO}_{2}$ substrates prior and after annealing were measured using a Ramé-Hart goniometer. $6 \mu \mathrm{L}$ of water was dropped on each sample using the automated drop volume control module and the static contact angle was measured with Droplmage software. 


\subsection{GIWAXS}

GIWAXS was performed at the CMS (11-BM) of the National Synchrotron Light Source II. Silicon wafers with a $100 \mathrm{~nm}$ layer of $\mathrm{TiO}_{2}$ were coated with pDA. Samples were exposed to a beam energy of $13.5 \mathrm{keV}(\lambda=0.9184 \AA)$ for 100 seconds at an incidence angle of $0.12^{\circ} .2 \mathrm{D}$ scattering patterns were obtained using a square Photonic Sciences CCD detector with a size of $1042 \times 1042$ pixels with pixel dimension of $0.1017 \mathrm{~mm} \times$ $0.1017 \mathrm{~mm}$. The sample-to-detector distance was $0.229 \mathrm{~m}$ with a detector tilt of $-21^{\circ}$ and detector orientation of $45^{\circ}$. Raw detector images were converted from $\left(q_{x}, q_{z}\right)$ to $\left(q_{r}, q_{z}\right)$ to account for Ewald sphere curvature ("missing wedge correction") using SciAnalysis, a python-based softwate developed by Brookhaven National Lab's CFN- Center for Functional Nanomaterials.1 Additionally, raw detector images analyzed using Xi-cam software (CAMERA at Lawrence Berkeley National Laboratory) to produce 1D intensity vs. $|q|$ plots in a line along the $q_{x}$ axis.2

\subsection{Scratch testing}

Nanoscratch tests were performed using Bruker's Hysitron TI-950 triboindenter equipped with a 2-D transducer and a $300 \mathrm{~nm}$ conospherical probe. The scratch load function consisted of three distinct segments: a trace segment to determine the surface profile at the site of the scratch, a 6 - $\mu \mathrm{m}$ long scratch segment, and a retrace segment to determine the residual deformation after the scratch. Two scratch test load functions were used: one with a constant normal load of $50 \mu \mathrm{N}$ applied during the scratch and the other with the normal load ramped linearly from 0 to $100 \mu \mathrm{N}$ during the length of the scratch. Multiple tests $(n=8)$ for each load function were performed on 3 samples of pristine and annealed pDA coatings in order to determine variation between films of the same type as well as between the two types of films tested. In addition, multiple tests $(n=8)$ for each load function were also performed on a $\mathrm{TiO}_{2}$ control sample. For all scratch tests, a tilt correction was performed on the scratch test data using the trace segment of the load function. Finally, using Hysitron's SPM imaging technique, the surface topography over an area of $15 \mu \mathrm{m} \times 15 \mu \mathrm{m}$ was mapped at each test site. 


\subsection{Nanoindentation}

Nanoindentation tests were performed using Bruker's Hysitron TI-950 triboindenter equipped with a 2-D transducer and a $300 \mathrm{~nm}$ conospherical probe. The tip area function was established from an aluminum standard. All load displacement curves were analyzed using the method described by Oliver and Pharr3 $30 \%$ of the unloading curve was used for analysis). All indentation tests were carried out in an open loop feedback mode under loading rate control. The peak load of approximately $25 \mu \mathrm{N}$ was used and was held fixed for a period of 3 seconds and load displacement curves were acquired. The slope of the load-displacement curve, upon unloading represents the stiffness, $\mathrm{S}$, evaluated at the maximum indentation depth $h_{\max }$. The stiffness was used to calculate reduced Young's Modulus Er using the relationship:

$$
E_{r}=\frac{\sqrt{\pi}}{2} \frac{S}{\sqrt{A_{c}}},
$$

With $A c$ being the contact area at $h_{\max }$. Finally, the reduced modulus can be expressed as a function of the elastic properties of the system (indenter and sample). Herein a Poisson's ration of $\mathrm{v}=0.35$, a typical value for macromolecules, was used.

\subsection{Single Molecule Force Spectroscopy (SMFS)}

Measurements were carried out using a JPK ForceRobot 300 (JPK Instruments AG, Germany) with a tip velocity of $1000 \mathrm{~nm} \cdot \mathrm{s}-1$ over a z-piezo distance of $500 \mathrm{~nm}$ with a dwell time of 1 second. The experiments were performed in UP water after allowing the cantilever to equilibrate in solution for at least 30 minutes. Soft silicon nitride cantilevers (MLCT from Bruker Nano Inc.) of typical spring constant of 50-60 pN.nm-1 were used for all experiments and calibrated using the equipartition theorem.4 The force-extension traces were recorded and analyzed using data processing software from JPK and a home-written procedure in IgorPro (Wavemetrics). 


\section{X-ray Photoelectron Spectroscopy (XPS)}

Table S1. Atomic concentration of functional groups in pDA and annealed pDA coatings obtained from the XPS measurements.

\begin{tabular}{|c|c|c|c|c|}
\hline \multicolumn{5}{|c|}{ C1s } \\
\hline & $\mathrm{CH}_{\mathrm{x}}, \mathrm{C}-\mathrm{NH}_{2}$ & $\mathrm{C}-\mathrm{O}, \mathrm{C}-\mathrm{N}$ & $C=0$ & $\pi-\pi$ \\
\hline pDA & $48.9 \pm 2.9$ & $32.5 \pm 2.0$ & $12.1 \pm 1.0$ & $6.4 \pm 1.4$ \\
\hline $\mathrm{pDA} 130^{\circ} \mathrm{C}$ & $50.2 \pm 1.4$ & $28.8 \pm 1.0$ & $15.9 \pm 0.9$ & $5.2 \pm 1.4$ \\
\hline \multicolumn{5}{|c|}{ O1s } \\
\hline & O-C & $O=C$ & & \\
\hline pDA & $64.2 \pm 3.3$ & $35.8 \pm 3.2$ & & \\
\hline $\mathrm{pDA} 130^{\circ} \mathrm{C}$ & $55.1 \pm 2.4$ & $45.1 \pm 2.7$ & & \\
\hline \multicolumn{5}{|c|}{ N1s } \\
\hline & $\mathrm{R}-\mathrm{NH}_{2}$ & $\mathrm{R}_{\mathbf{2}}-\mathrm{NH}$ & $=\mathbf{N}-\mathbf{R}$ & \\
\hline pDA & $14.6 \pm 0.8$ & $78.8 \pm 0.6$ & $6.6 \pm 0.8$ & \\
\hline pDA $130^{\circ} \mathrm{C}$ & $9.5 \pm 0.5$ & $84.9 \pm 0.8$ & $5.6 \pm 0.7$ & \\
\hline
\end{tabular}




\section{Contact Angle Measurements}

The increase in the contact angle after thermal treatment may be ascribed to the disappearance and transformation of polar groups in the pDA structure. XPS spectra show transformation of phenolic hydroxyls to quinones ( $25 \%$ increase in content) as well as a significant reduction in the primary amine content. The increase in the carbonyl content after thermal treatment has been previously utilized to increase the bioconjugation capacity of the pDA coatings.5-6

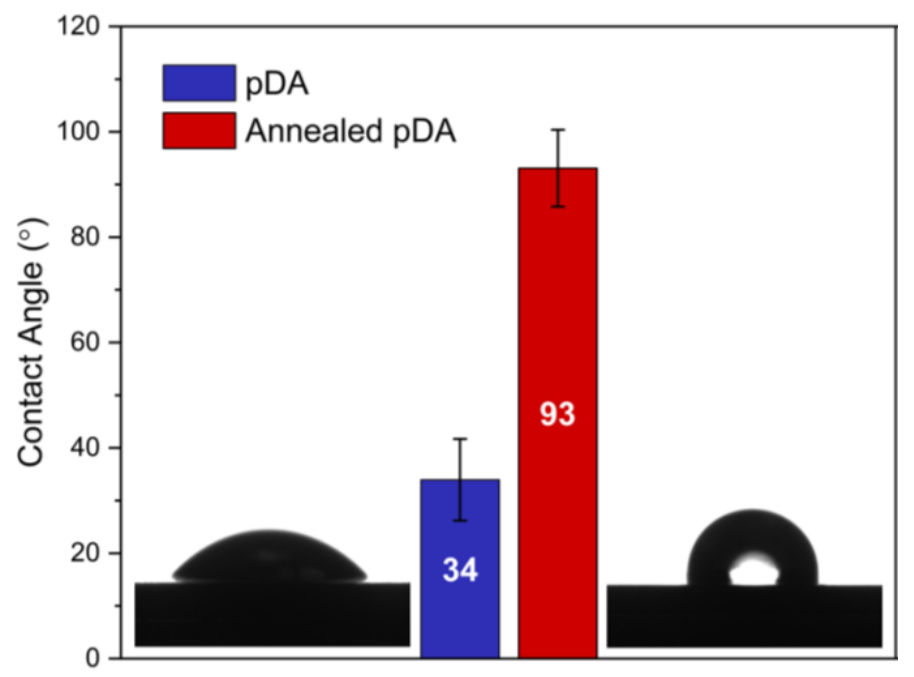

Figure S1. Water contact angle of the pristine pDA and annealed pDA coatings, showing a drastic increase in the surface hydrophobicity after annealing. Error bars represent standard deviation of 15 independent measurements. 


\section{UV-Vis Absorbance Spectrum}

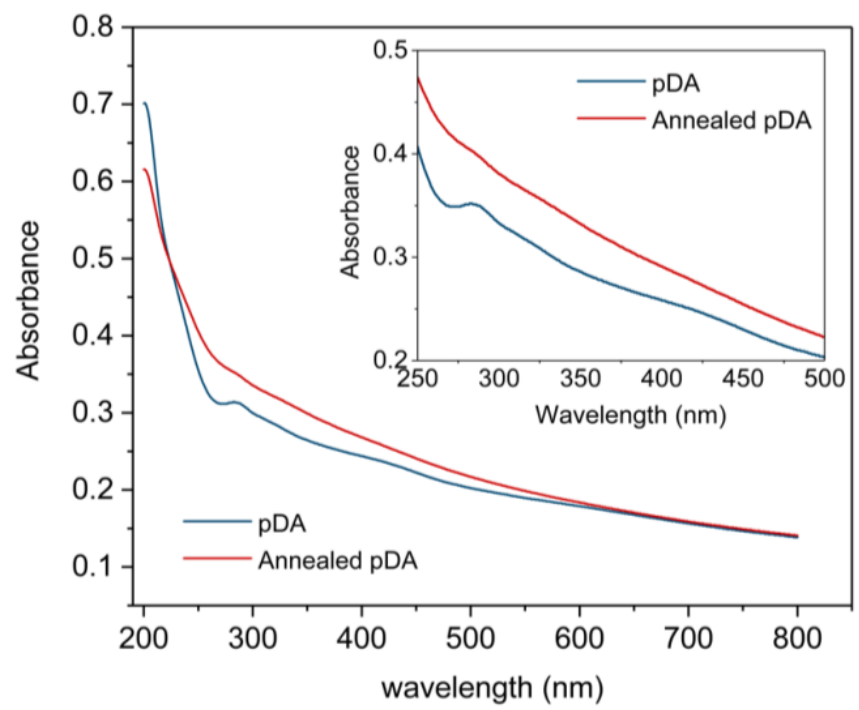

Figure S2. UV-Vis spectra of the pDA-coated quartz slides before and after thermal annealing at $130{ }^{\circ} \mathrm{C}$. The peak at $280 \mathrm{~nm}$ and the shoulder at $420 \mathrm{~nm}$ in the blue curve correspond to the fraction of monomeric and oligomeric species present in the pristine pDA coating. After annealing, the intensity of these features decreases significantly, indicating the conversion and further oxidation of partially polymerized species resulting in a monotonous absorption behavior. Inset shows a zoom-in of the spectrum from 250 to $500 \mathrm{~nm}$. 


\section{Thickness Evaluation after Sonication}

One the main drawbacks of pDA coatings is their weak stability under sonification. Pristine pDA and annealed pDA coated on $\mathrm{TiO}_{2}$ were sonicated in an aqueous environment ( $\mathrm{pH} 6.9$ ) at high power for 10 minutes. After sonication, the thickness of pDA dropped by $\sim 35 \%(n=9, p<0.05)$ whereas the thickness of the annealed pDA did not show any statistically significant reduction. After thermal annealing the stability of pDA against sonication was enhanced possibly due to the enhancement in the intermolecular and cohesive interactions between pDA chains.

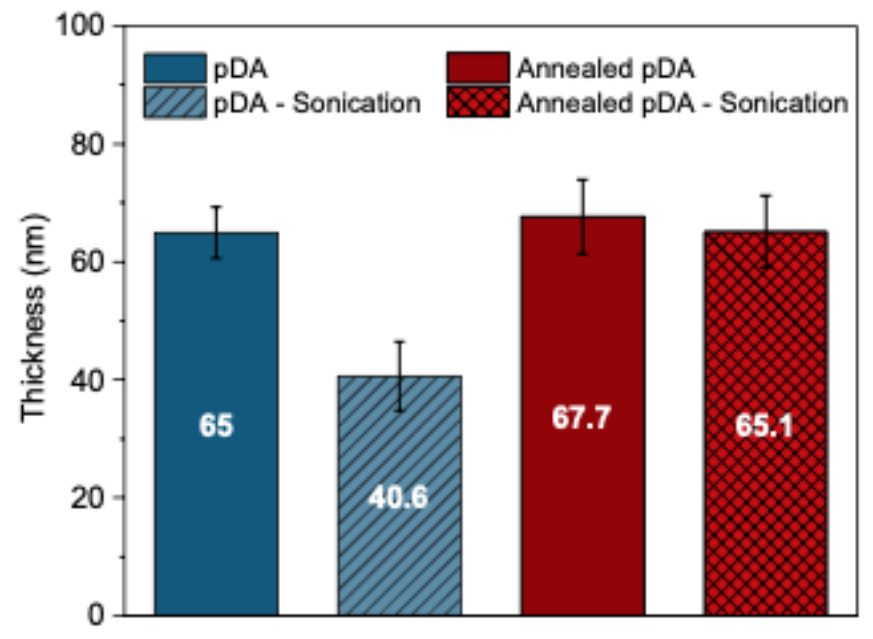

Figure S3. Ellipsometry measurements showing changes in thickness of the pristine and annealed pDA coatings prior to and after sonication in Milli-Q water for 10 minutes. 


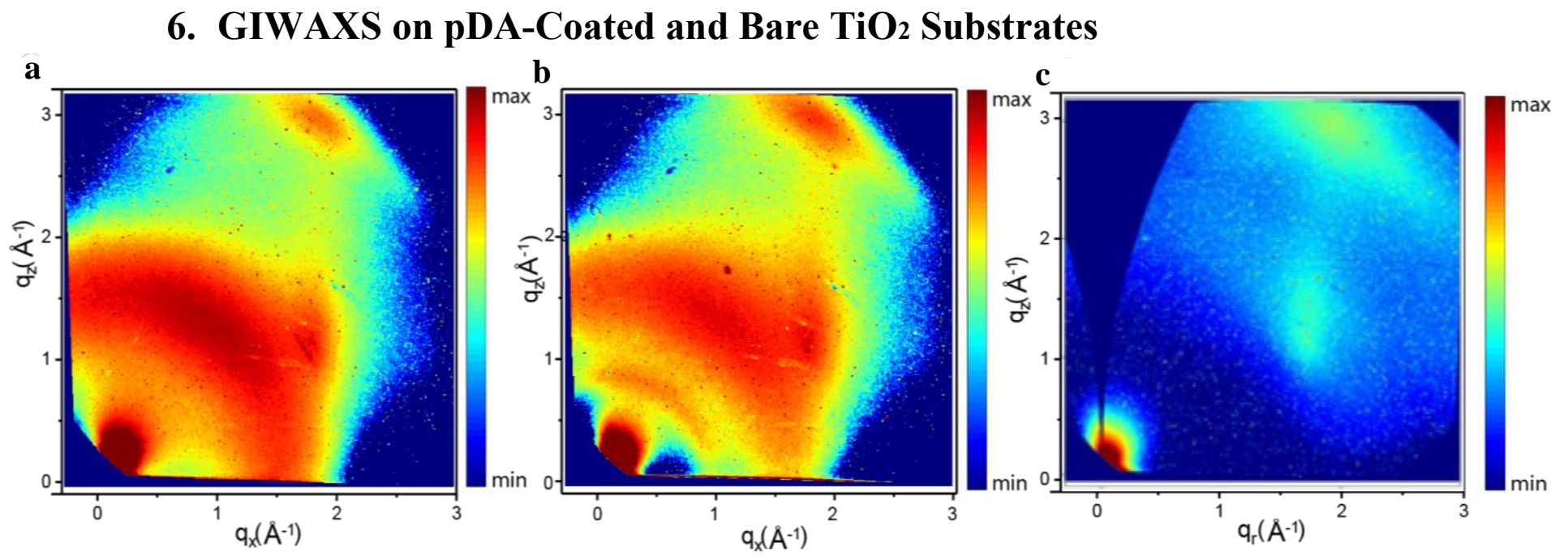

Figure S4. Raw 2D GIWAXS detector images of pristine $\mathrm{pDA}$ coatings on $\mathrm{TiO}_{2}$ (a) and annealed pDA coatings on $\mathrm{TiO}_{2}$ (b). 2D GIWAXS image of bare $\mathrm{TiO}_{2}$ after missing wedge correction (c). No phase transformational changes occur during thermal annealing of $\mathrm{TiO}_{2}$ at $130 \circ \mathrm{C} .7-8$ 


\section{Scratch Resistance}

\subsection{Constant Load $-50 \mu N$}

a

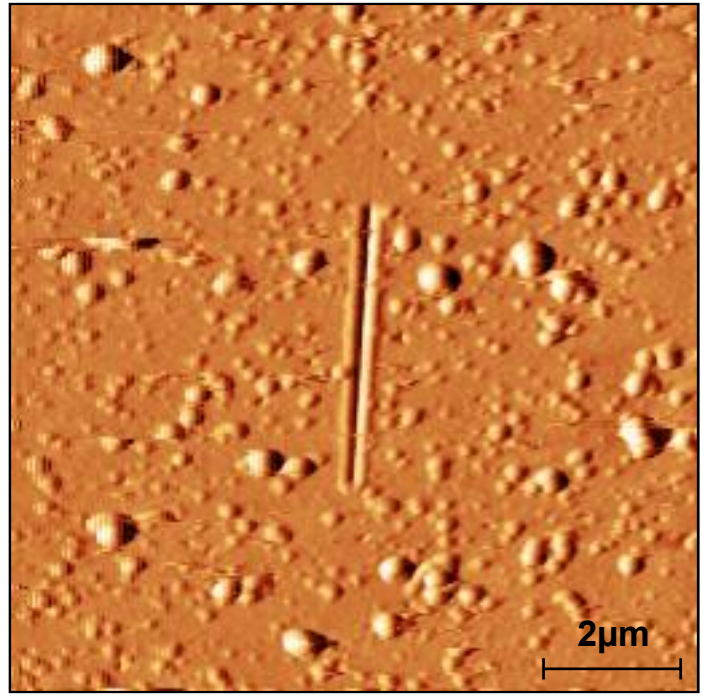

b

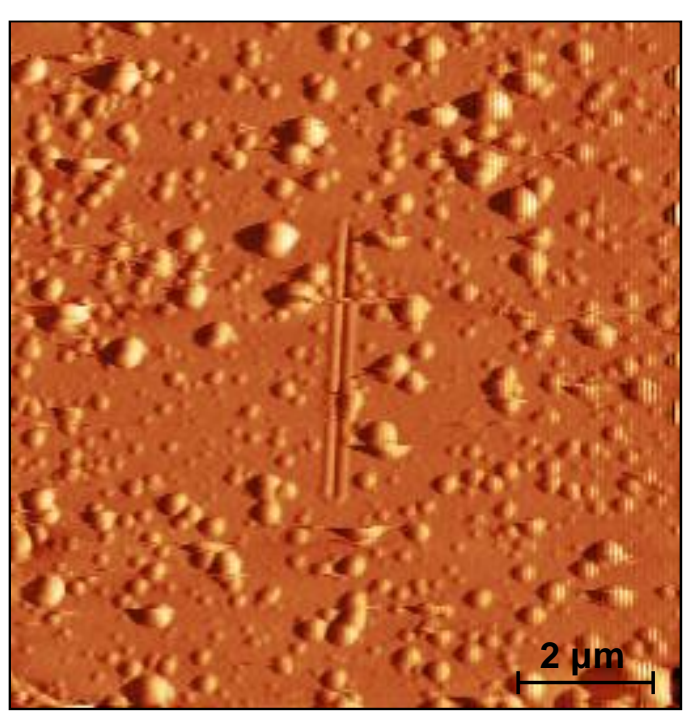

Figure S5. Images of scratches on the pristine pDA (a) and annealed pDA (b) using a 50 $\mu \mathrm{N}$ constant load. An improvement in the scratch resistance is noted for the annealed pDA, indicating the enhanced ability of the coating to withstand the load resulting in smaller surface deformation.

\subsection{Progressive Load $-\mathbf{1 0 0} \mu \mathrm{N}$}

The influence of the scratch load on the mechanical response of the pDA coatings prior to and after annealing was investigated by carrying out progressive load scratch measurements. This second set of the scratch tests, where the normal force was ramped from 0 to $100 \mu \mathrm{N}$ during the scratch, were performed in order to induce failure of the film to compare critical loads at failure between the two sets of films. The normal displacement during the scratch segment as well as that during the retrace segments for the ramp load scratch test is shown in Figure S6. No evidence of cracking or delamination failure was observed in either set of films up to $100 \mu \mathrm{N}$ normal force. However, the scratch depth was equal to or greater than the film thickness for most tests on all pristine samples tested. The penetration depth was lower while the recovery after load removal was higher for the annealed pDA coatings compared to pristine coatings evidence of the higher penetration resistance. After thermal annealing, pDA films show higher resistance to scratch as 
compared to pristine pDA films, consistent with the expectation that the annealing process increases the cross-linking of the polymeric chains and cohesion of pDA films, resulting in a robust coating with superior mechanical properties.
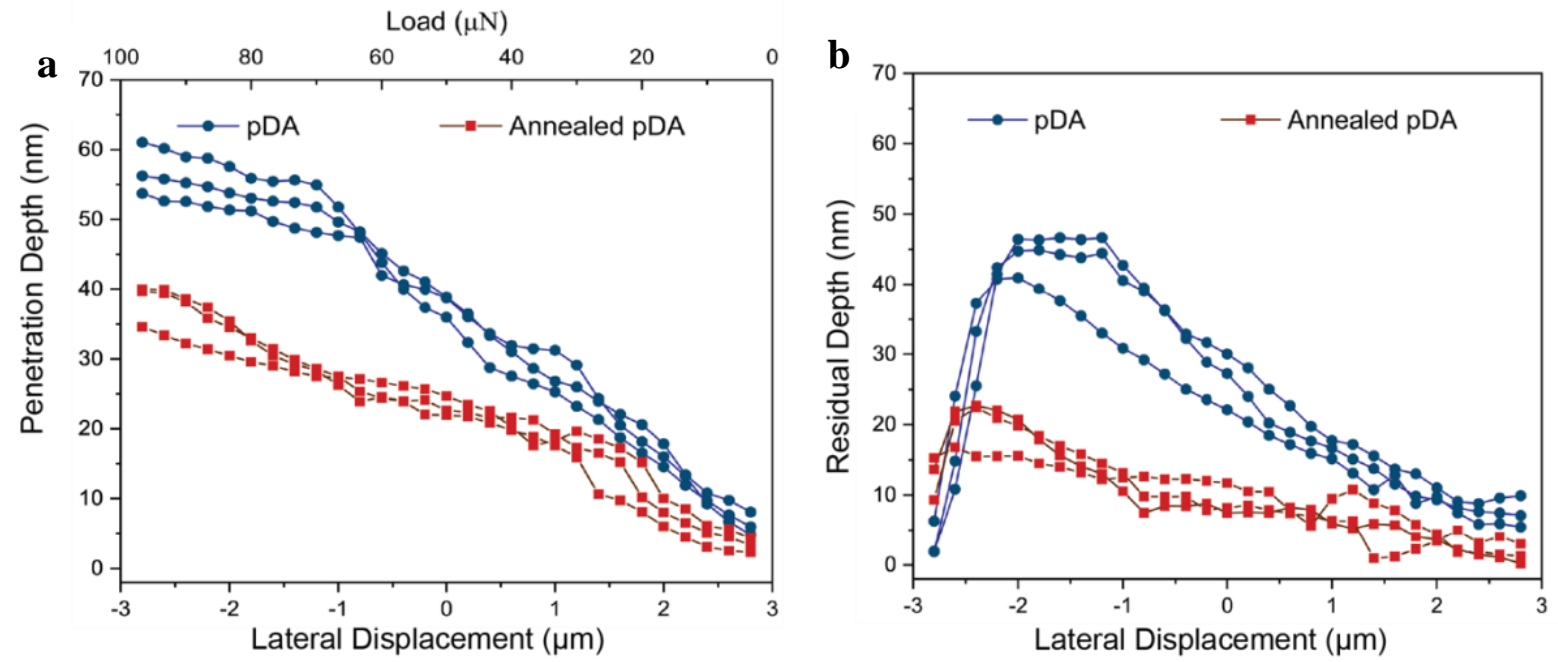

c

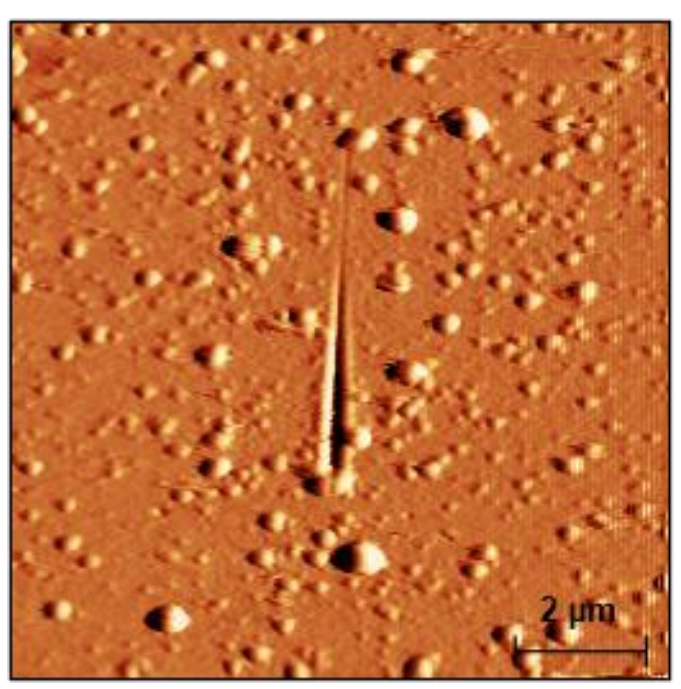

d

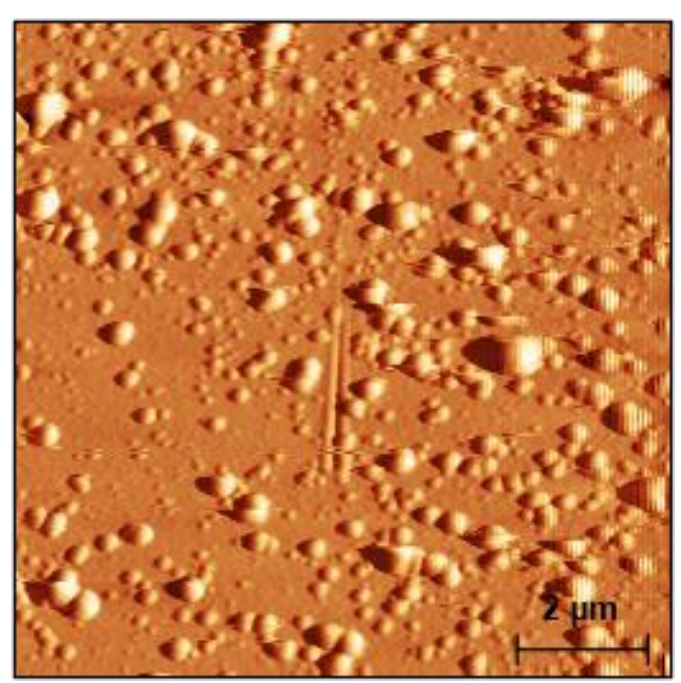

Figure S6. Scratch resistance measurements on the pristine and annealed pDA coatings using a progressive load up to $100 \mu \mathrm{N}$. Representative penetration-lateral displacement and residual depth-lateral displacement curves during scratching shown in (a) and (b), respectively. Images of the scratches on the pristine pDA (c) and annealed pDA (d) during the $100 \mu \mathrm{N}$ progressive load. 


\section{Elastic Modulus}

The elastic modulus of pristine pDA was measured $\sim 2.8 \pm 0.8 \mathrm{GPa}$ whereas after thermal annealing the modulus increased to $\sim 7.4 \pm 1.2 \mathrm{GPa}$. Thermal annealing resulted in a significant increase on the elastic modulus due to the enhancement in the intermolecular and cohesive interactions between the chains which resulted in mechanical interlocks that enhanced the resistance to elastic deformation. Similar increase in the elastic modulus of pDA films has been observed before and attributed to the enhanced cross-linking. For instance, Klosterman et al. demonstrated that chemical cross-linking of the pDA films using Genipin molecules can result in almost 5 times increase in the modulus from $1.5 \pm 0.4$ to $7.9 \pm 1.7 \mathrm{GPa} .9$ More recently $\mathrm{Li}$ et al. showed that heat treatment of pDA films at elevated temperatures $\left(300\right.$ to $\left.600{ }^{\circ} \mathrm{C}\right)$ can result in enhancing the Young's modulus of the films from $2.3 \pm 0.84 \mathrm{GPa}$ to $\sim 7 \mathrm{GPa}$ after treatment at $300{ }^{\circ} \mathrm{C}$ and $\sim 14 \mathrm{GPa}$ when treated at $600{ }^{\circ} \mathrm{C} .10$

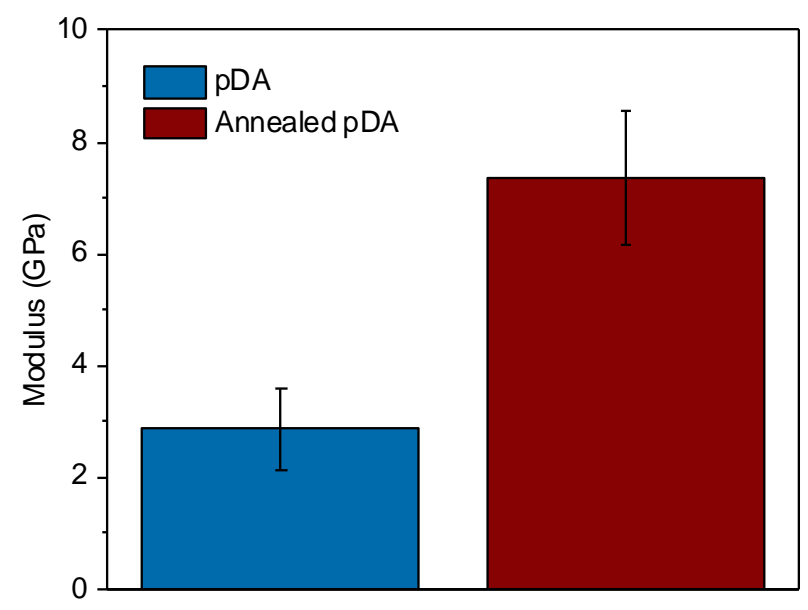

Figure S7. Elastic modulus determined by nanoindentation on pristine and annealed pDA. 


\section{9. pDA-Assisted Electroless Metallization}

The metal binding ability of catechols in the pDA coating is known to assist the deposition of an adherent and uniform metal film onto substrates by electroless metallization. Here the electroless silver metal film deposition via dip coating of pDA and annealed pDA coated $\mathrm{TiO}_{2}$ substrates into silver nitrate solution was investigated. As Figure $\mathbf{S 8}$ shows after thermal annealing the apparent reductive capacity of pDA layer was sufficient to eliminate the need for addition of a reducing agent implying the ability of the annealed $\mathrm{pDA}$ layer to reduce metal ions comparable to that of the pristine $\mathrm{pDA}$.
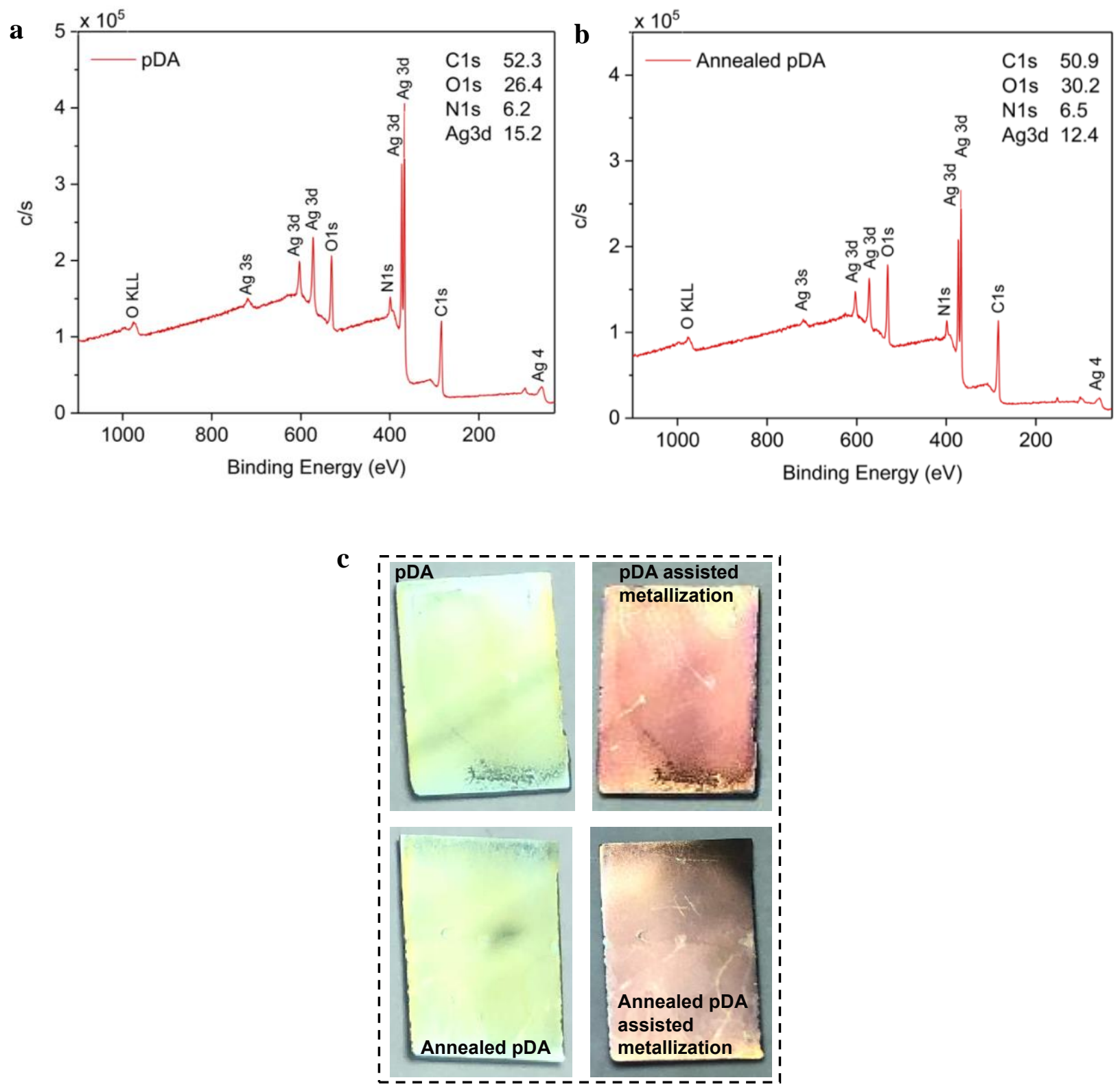
Figure S8. XPS spectra on pDA (a) and annealed pDA (b) - assisted electroless metallization of $\mathrm{pDA}$ coated $\mathrm{TiO}_{2}$ substrates. Representative images of $\mathrm{pDA}$ and annealed pDA coated $\mathrm{TiO}_{2}$ substrates prior to (left) and after (right) the electroless metallization (c).

\section{References}

(1) GitHub SciAnalysis. https://github.com/CFN-softbio/SciAnalysis.

(2) Pandolfi, R. J.; Allan, D. B.; Arenholz, E.; Barroso-Luque, L.; Campbell, S. I.; Caswell, T. A.; Blair, A.; De Carlo, F.; Fackler, S.; Fournier, A. P.; Freychet, G.; Fukuto, M.; Gursoy, D.; Jiang, Z.; Krishnan, H.; Kumar, D.; Kline, R. J.; Li, R. P.; Liman, C.; Marchesini, S.; Mehta, A.; N'Diaye, A. T.; Parkinson, D. Y.; Parks, H.; Pellouchoud, L. A.; Perciano, T.; Ren, F.; Sahoo, S.; Strzalka, J.; Sunday, D.; Tassone, C. J.; Ushizima, D.; Venkatakrishnan, S.; Yager, K. G.; Zwart, P.; Sethian, J. A.; Hexemer, A. Xi-cam: a versatile interface for data visualization and analysis. J Synchrotron Radiat 2018, 25, 1261-1270.

(3) Pharr, G. M.; Oliver, W. C.; Brotzen, F. R. On the Generality of the Relationship among Contact Stiffness, Contact Area, and Elastic-Modulus during Indentation. J Mater Res 1992, 7 (3), 613-617.

(4) Lübbe, J.; Temmen, M.; Rahe, P.; Kühnle, A.; Reichling, M. Determining cantilever stiffness from thermal noise. Beilstein Journal of Nanotechnology 2013, 4, 227--233.

(5) Martin, M.; Orive, A. G.; Lorenzo-Luis, P.; Creus, A. H.; Gonzalez-Mora, J. L.; Salazar, P. Quinone-Rich Poly(dopamine) Magnetic Nanoparticles for Biosensor Applications. Chemphyschem 2014, 15 (17), 3742-3752.

(6) Luo, R. F.; Tang, L. L.; Wang, J.; Zhao, Y. C.; Tu, Q. F.; Weng, Y. J.; Shen, R.; Huang, N. Improved immobilization of biomolecules to quinone-rich polydopamine for efficient surface functionalization. Colloid Surface B 2013, 106, 66-73.

(7) Eastman, J. A. Microstructural Development in Nanophase Tio2 during Annealing. J Appl Phys 1994, 75 (2), 770-779.

(8) Rath, H.; Anand, S.; Mohapatra, M.; Dash, P.; Som, T.; Singh, U. P.; Mishra, N. C.; Kanjilal, D.; Avasthi, D. K. Phase Transformation of TiO2 from Anatase to Rutile by Thermal Annealing and Swift Heavy Ion Irradiation. Aip Conf Proc 2008, 1063.

(9) Klosterman, L.; Ahmad, Z.; Viswanathan, V.; Bettinger, C. J. Synthesis and Measurement of Cohesive Mechanics in Polydopamine Nanomembranes. Adv Mater Interfaces 2017, 4 (10).

(10) Li, H. Q.; Xi, J. X.; Zhao, Y.; Ren, F. Mechanical properties of polydopamine (PDA) thin films. Mrs Adv 2019, 4 (7), 405-412. 